\title{
A method for estimating the number of motor units in thenar muscles and the changes in motor unit count with ageing
}

\author{
W. F. BROWN
}

From St. Joseph's Hospital, London, Ontario, Canada

SUMMARY A method for estimating the number of motor units in median innervated thenar muscles is described. In normal human subjects under 40 years of age, the mean and 1 SD was $253+34$. The number of motor units falls with increasing age, especially after mid life, so that elderly subjects often have less than one-half the number in youth despite good strength. The cause is not known but probably includes peripheral nerve trauma and 'primary neuronal cell death'.

Recent electrophysiological evidence for a postulated neurogenic basis of several muscular dystrophies, including dystrophia myotonica (McComas, Campbell, and Sica, 1971b), limb-girdle and fascioscapulohumeral dystrophy (Sica and McComas, 1971) and Duchenne type muscular dystrophy (McComas, Sica, and Currie, 1971), was based on a method for counting and estimating the size of motor units in the extensor digitorum brevis muscle (McComas, Fawcett, Campbell, and Sica 1971a). This muscle was chosen because of its isolation from other muscles, the single end-plate zone, relatively thin overlying skin, and the fact that it was a flat muscle and the motor units were likely to be scattered evenly throughout the muscle.

The conclusions of the authors may be criticized because of possible inadvertent and asymptomatic injury to the peroneal nerve, although slowing of conduction and prolongation of the terminal transmission delay was not found (McComas et al.,1971b). The technique was a sensitive method for demonstrating denervation. McComas has stated that abnormally low values were always found in patients with clinical or electrophysiological evidence of denervation and, further, in some patients in whom the electromyographic interference pattern appeared normal, denervation was readily shown by the motor unit count estimate (McComas et al., 1971a).

The deliberate choice of the extensor digi- torum brevis muscle provided the most reasonable recording conditions in normal subjects for motor unit counts. Despite the technique's undoubted value as a sensitive quantitative method for demonstrating denervation, several important neurological diseases affect the upper limb exclusively or as an early feature. For this reason, the present study was undertaken in an attempt to provide a feasible reproducible estimate of the motor unit count in selected small muscles of the hand.

The method to be described is relatively simple, requires a minimum of patient cooperation and provides a quantitative estimate of the numbers of motor axons and motor units. The approximations and many of the assumptions are similar to the parent study of the extensor digitorum muscle by McComas (McComas et al., 1971a).

Single axons in a motor nerve have a critical threshold for all-or-nothing response to an electrical stimulus. Activation results in a compound action potential from all of the muscle fibres innervated by that axon. Provided the conduction velocity variation in motor axons to a muscle does not vary greatly and the end-plate zone for individual motor units is located equidistant from the recording electrode, successively recruited individual motor unit potentials will sum in a linear way. It is possible, by carefully grading the stimulus to a motor nerve to count clearly 
the first five to 15 motor unit steps. The compound potential amplitude of the first several potentials divided by the number of steps, gives an estimate of average motor unit size and this value divided into the total compound action potential with a supramaximal stimulus to the motor nerve, provides the estimated motor unit count.

This paper describes the method and results in a series of normal patients. Papers to follow will discuss the application of the technique to a variety of neurogenic disorders affecting the upper limbs, including the carpal tunnel syndrome, and to a study of the late responses from human hand muscles.

\section{METHODS}

There were 44 subjects between the ages of 13 and 89 years, 14 of whom were female. The controls came from members of the staff, medical students, and patients with no history or findings of neurological abnormality in the arm. Specifically, no patient was included with any history of sensory or motor symptoms in the hand or arm.

PREPARATION The belly of the abductor pollicis brevis muscle can be palpated easily as a separate mass when the thumb is abducted against resistance. In no patient in this series was this muscle found to be supplied by the ulnar nerve.

RECORDING The recording electrode was placed in the mid-belly position and from this point the initial deflection of the action potential was always negative, whether with successive voluntarily evoked single motor units or the first several units recruited with median nerve stimulation. Movement of the recording electrode, even a few millimetres distal or proximal, often caused the initial deflection to be positive, providing evidence that the end-plate zone was located in the mid-belly position of the abductor pollicis brevis muscle.

A variety of electrodes were tried but the most satisfactory were found to be stainless steel surface clip electrodes (Copland-Davies, Electrophysiological Instruments Limited, Edinburgh, Scotland). The active electrode was placed over the mid-belly position of the abductor pollicis brevis muscle or moved slightly so that the initial deflection of motor unit potentials, evoked by thumb abduction and median nerve stimulation, was negative and the amplitude of the negative deflection with supramaximal stimulation to the median nerve was maximum. The reference electrode was placed at the base of the proximal phalanx where the tendon of the abductor pollicis brevis muscle is inserted (Davies and Coupland, 1967). The arm was secured firmly by rubber straps to a padded board. One of these straps was used to secure the forearm proximal to the stimulating electrodes and the other, the hand, including the thumb, in the adducted position. These precautions were taken to minimize movement. The hand was earthed by a platinum gauze electrode, wetted with electrode paste and held securely over the dorsum of the hand, although in some instances where the stimulus artefact was a problem, the earth electrode was changed $\stackrel{5}{9}$ to a platinum strip and held in place at the base of $\bar{C}$ the thumb between the stimulating electrode and the recording electrodes.

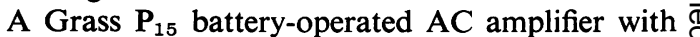
frequency limits between $0 \cdot 1$ to $3 \mathrm{db}$ at $3 \mathrm{kHz}$ was used. The input impedance of this amplifier was $200 \mathrm{M} \Omega$ differential. With the input shorted the noise was. $3 \mu \mathrm{V}$ and with the electrodes in place on the subject, the peak to peak noise was no more than $5 \mu \mathrm{V}$.

STIMULATION A DISA bipolar surface electrode (13K62) was used to stimulate the median nerve at $\omega_{\mathrm{w}}$ the wrist. This electrode was held securely in place $\sigma$ by a plastic band and the nerve stimulated at frep quencies of less than $1 \mathrm{sec}$.

The stimulus was delivered by a Devices Mode 3072 high voltage stimulator with pulses of usual 50 to $100 \mu \mathrm{sec}$ in duration. The stimulus voltage was slowly and smoothly increased several times to certain that all potential steps were clearly recos 3 nized and the step potential changes superimposed. 은 The possibility of stimulating two or more axom with similar thresholds and failing to recognize the separate single axon potential steps was minimized by repeating the stimulus sequence.

DISPLAY The data were displayed and stored on a Tektronix 5103N oscilloscope and permanent records $\stackrel{2}{\circ}$ taken with a Polaroid camera. In some of the early $\mathbb{\complement}$ studies, a Tektronix 565 oscilloscope was used and $\overrightarrow{\vec{O}}$ superimposed traces photographed by a Grass Model 3 C4 camera.

\section{RESULTS}

The results in two representative control subjects: of different ages are shown in Fig. 1. The records? on the left show the results with near threshold 0 and supramaximal stimulation of the median nerve in a 23 year old male. The average motor unit size, based on the first 11 potential steps, $\frac{D}{O}$ was $42 \mu \mathrm{V}$. With supramaximal stimulation of the median nerve at the wrist the size of the maxi- $N$ 


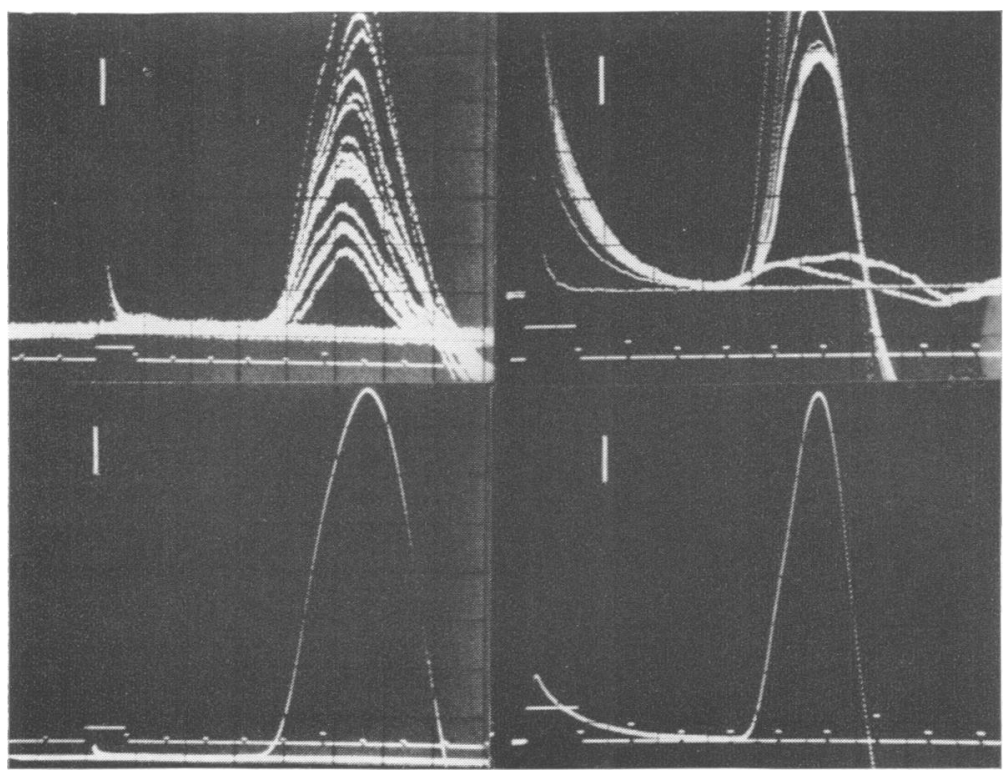

FIG. 1. The motor unit count records from the 23 year old student are shown on the left. The records on the right are from a 70 year old man. The upper records show the first few potential steps (Cal. 100 $\mu V)$ and the lower traces the maximum compound potentials (Cal. R.L. $1 \mathrm{mV}$, L.L. $2 \mathrm{mV}$ ).

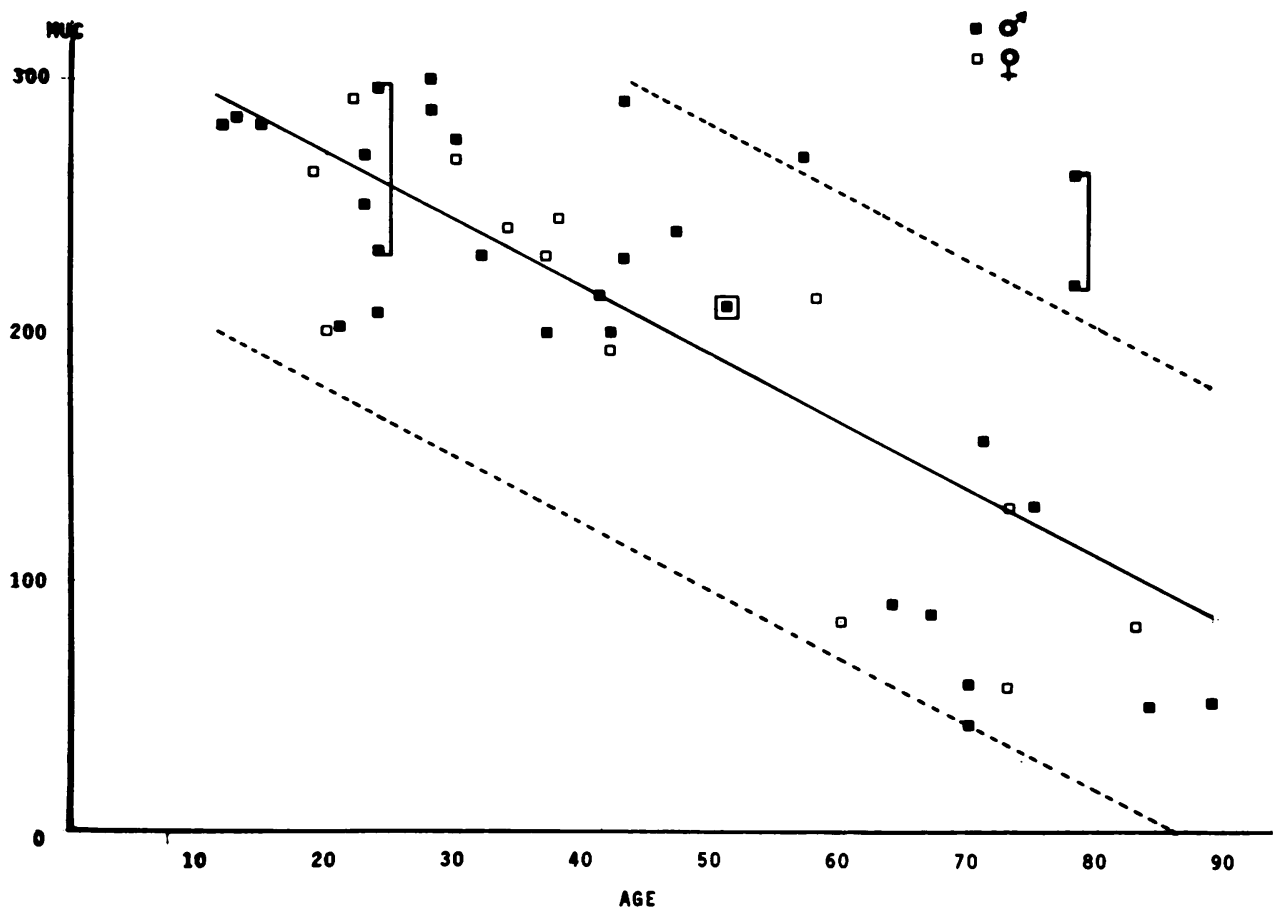

FIG. 2. Plot of motor unit count against age. Regression line shown \pm 2 SE. Vertical bars connect values in both hands of the same patient. 
mum negative deflection from base-line to peak was $15.4 \mathrm{mV}$ and based on this value and the average motor unit size the estimated motor unit number was 248 . On the right, the results in a 70 year old male are shown. In this man, the estimated number of motor units was 60 . Only the first five steps are shown in the accompanying photograph.

In this study, the potential amplitudes were measured from the baseline to the negative peak instead of from peak to peak. The differences in the count figures were never greater than $5 \%$ using the two methods, and the method in this study allowed more careful definition of the potential steps.

A plot of the motor unit estimates of all the control subjects is shown in Fig. 2.

The estimate of motor unit count was relatively independent of the recording situation, provided that successive increments of amplitude could be recognized satisfactorily. The estimate of the average motor unit size is less certain because it is more dependent on the recording situation (McComas et al., 1971a). Moreover, the study was further complicated by the fact that the motor units in the thenar muscle mass are not randomly distributed but confined to the individual muscles. Even if motor units in the abductor pollicis brevis muscle were the same size as those, for example, in the opponens pollicis muscle, the latter would appear smaller because of their greater distance from the recording electrode.

The numbers of large diameter nerve fibres to the first lumbrical and first dorsal interosseous muscles have been counted and, based on the assumption that $60 \%$ of the large diameter nerve fibres were efferent, an estimate of the numbers of motor units in these muscles was made (Feinstein, Lindegaard, Nyman, and Wohlfart, 1955). The $60 \%$ proportion was in turn based on earlier work in the cat (Eccles and Sherrington, 1930) and one human case of poliomyelitis (Feinstein, et al., 1955). There is no good reason to think that the $60 \%$ is more than roughly correct but, based on it, Feinstein estimated the number of motor units in the human first lumbrical muscle to be 93 in a 54 year old subject and 98 in a 29 year old subject.

The estimated number of motor units in this study (Figs 1 and 2) was more than twice that estimated for the first lumbrical muscle, but it is possible to reach some estimate of the number of motor units in the thenar muscle by comparing $\mathbb{\mathscr { D }}$ spindle counts in various hand muscles.

The results of muscle-spindle counts in a variety of human muscles, including a number of small hand muscles, were collected and listed by Cooper (1960). There were, for example, $80 \stackrel{\text { }}{工}$. spindles in the abductor pollicis brevis, 44 in the $\stackrel{\vec{F}}{\overrightarrow{7}}$ opponens pollicis, and 27 in each of the two. heads of the flexor pollicis brevis muscles and $\overrightarrow{\vec{F}}$ for comparison, 51 in the first lumbrical muscle. $\overrightarrow{0}$ If, as an approximation, it is assumed that the $\frac{}{0}$ proportion of muscle spindles to motor units is $\frac{\bar{\sigma}}{\frac{5}{\sigma}}$ similar in the small muscles of the hand, then the $\stackrel{\mathbb{Q}}{\circ}$ estimated number of motor units in the abductor pollicis brevis and opponens pollicis muscles $\vec{\circ}$

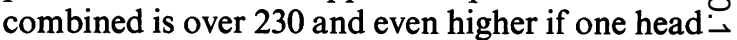
of the flexor pollicis brevis muscle is included. $\vec{\omega}$ These estimates are within the range of the? estimated number in the study.

The recording method precluded a confident identification of the thenar muscle of origin of $\%$ any particular motor unit step potential in most instances. The initial deflection was always pos tive with the recording electrodes in the standaed 5 position when the ulnar nerve was stimulated. $\stackrel{\unrhd}{\varrho} \overrightarrow{0}$

The scatter of motor unit numbers (Fig. even in the younger subjects, was probably the result in part, of a variation in nerve supply the thenar muscles. In no instance in this studo was the abductor pollicis brevis muscle found to be innervated by the ulnar nerve. No other detail-? ed studies looking for evidence of anomalous innervation of the thenar muscles using local anaesthetic block of the ulnar or median nerves or electromyographic methods, was done in this

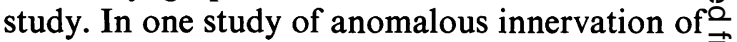
the hand muscles, the flexor pollicis brevis $\overline{\bar{B}}$ muscle was supplied by the ulnar nerve exclu- $\frac{3}{5}$ sively in $32 \%$ and split innervation between the median and ulnar nerves in $15.5 \%$. In the remainder, this muscle was wholly supplied by the median nerve (Rowntree, 1949). In that study: of 124 complete ulnar nerve interruptions, only one case was found where the opponens pollicis muscle was paralysed.

The motor unit count estimate with this method was based on the average motor unit $>$ size of the first 2 to $5 \%$ of axons activated. Kugelberg showed that the larger spike poten- $N$ 
tials in the first dorsal interosseous muscle had distinctly higher thresholds to voluntary activation as well as to electrical stimulation of the ulnar nerve (Kugelberg and Skoglund, 1946). The study of the motor unit population in the extensor digitorum communis muscle of the baboon forearm, showed that fewer than $10 \%$ of the motor units had twitch tensions over twice the size of the far more numerous smaller units, (Eccles and Phillips, 1968). The question of how representative the first several motor unit potentials were of the whole population of motor units has previously been discussed in respect to the extensor digitorum brevis muscle (McComas et al., 1971a). In the present study-for example, Fig. 1-there was a variation of motor unit size but no consistent progression to motor units of larger size with mounting stimulus intensities. Frequently, in the first three to five step potentials there was one particularly large unit. Voluntary activation of motor units from the thenar group does recruit motor units, however, of progressively larger action potential size and it is possible that our motor unit count is an overestimate.

Another source of an over-count of motor units is variable sequence summing of motor units. This is most likely to happen if the motor axons have closely similar thresholds. If two or more motor axons are always activated together because of similarly critical thresholds, the motor unit estimate may be falsely low. If, however, their thresholds are similar and repeated efforts made to separate them, the order of summing of individual motor unit potentials may change from stimulus sequence to sequence with the result that a number of the motor units are overestimated. These are some of the main reasons for limiting the average motor unit size calculation to the first 2 to $5 \%$ of the units that can clearly be distinguished.

The method also depends on linear summation of individual motor unit potentials in an orderly recruitment sequence. If the terminal delay to individual motor units varies so that there is a temporal dispersion of motor unit potentials, summation will not be linear and the result is that the estimated motor unit potential size is falsely low and the motor unit count number too high.
Temporal dispersion, as a factor in the motor unit count, was more of a problem in the abnormal cases and its effect will be discussed in more detail in a later study of motor unit counts in patients with carpal tunnel syndrome.

The motor unit count should more strictly be considered an axon unit count because of the possibility of peripheral axon branching at or proximal to the site of stimulation of the median nerve at the wrist. Gilliatt did not find any evidence of axon reflexes in the median or ulnar nerves in normal subjects (Gilliatt, 1966) but evidence for axon branching was found by Stålberg in the tibial nerve trunk, as high as 100 to $150 \mathrm{~mm}$ above the motor point in young healthy subjects (Stålberg and Trontelj, 1970). Wray has shown that in the baboon nerve to the abductor pollicis brevis muscle, between 32 and $22 \mathrm{~mm}$ from the motor point, the percentage increase in branching varied in two animals between 7 and $21 \%$ (Gilliatt, 1966).

In a few of the present subjects a motor unit estimate was done at the elbow and the wrist. The motor unit count was in reasonable agreement at the two levels, although the average motor unit potential size was usually smaller at the proximal stimulation site compared with the wrist, probably because of mild temporal dispersion of motor unit potentials (Hopf, 1962). The effect of possible increase in axon branching with ageing would have been to make the motor unit estimates higher than they should be and, in the older ages, closer to the younger control subjects.

In Fig. 3 the graph shows the average motor unit potential size plotted against the estimated motor unit numbers.

In this study, the average motor unit potential size in all subjects 59 years old and under was $53 \mu \mathrm{V} \pm 7.91 \mathrm{SD}$ compared with a mean and $1 \mathrm{SD}$ of all those controls with motor unit counts of 100 and less, of $100 \pm 51 \mu \mathrm{V}$. Despite the obvious scatter and several subjects with low motor unit counts but motor unit potential sizes in the normal range, there were others where the motor unit potential size was clearly much enlarged. All of the subjects regardless of motor unit potential size had full strength as tested in the conventional clinical manner, although the maxi- 


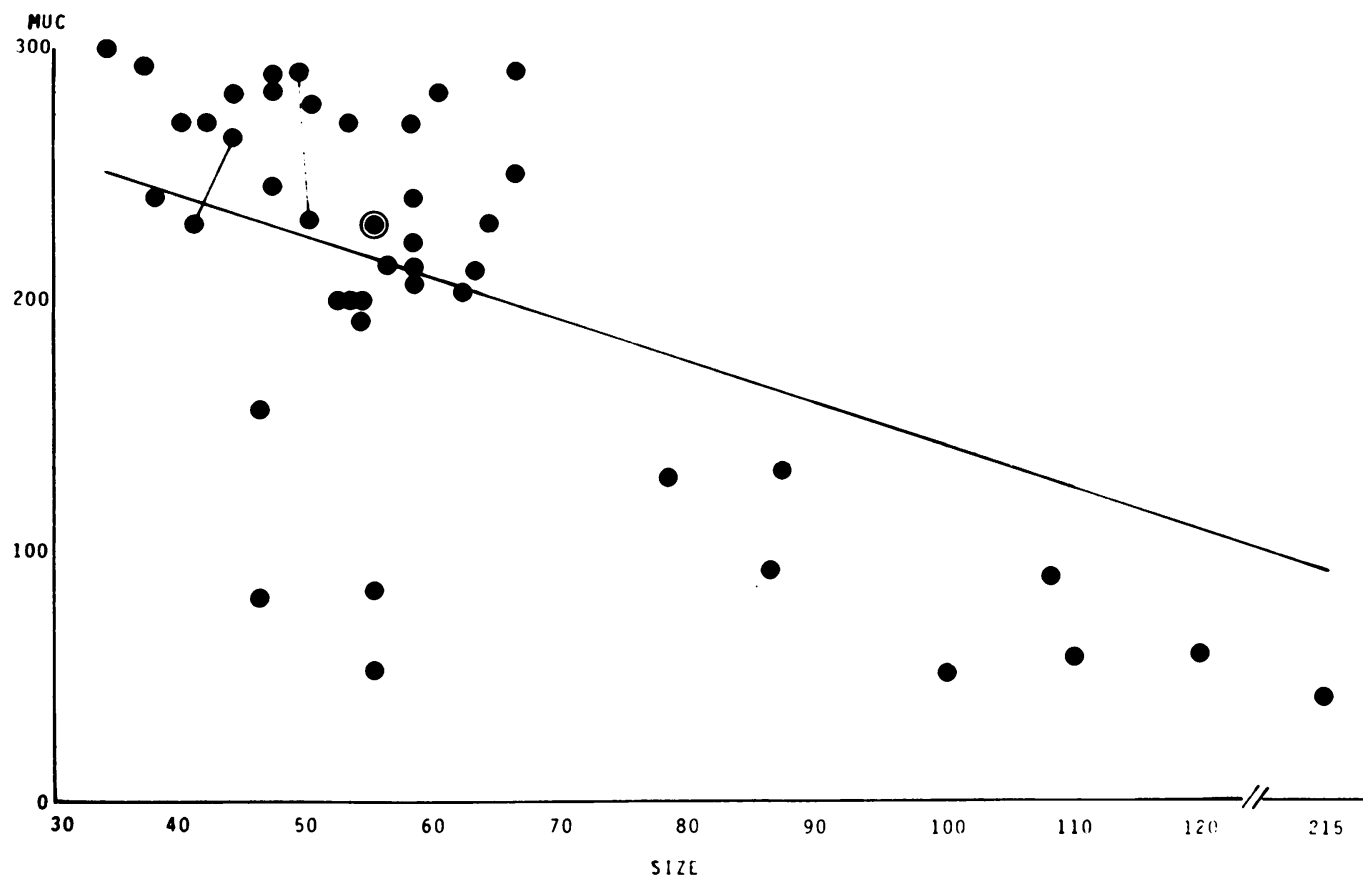

FIG. 3. Plot of motor unit count against motor unit potential size including the regression line. Vertical bars connect values in both hands of the same patient.

mum twitch tensions were not measured. There was no significant difference between the males and females in motor unit size or number.

\section{DISCUSSION}

The present study has shown a remarkable fall in the number of motor units in the thenar muscles supplied by the median nerve, especially in the subjects over 60 years old. Severe denervation in the extensor digitorum brevis muscle had previously been shown by Campbell (Campbell and McComas, 1970), in many patients over 60 years. Despite a considerable reduction in the number of motor units, strength assessed clinically was maintained. This study provided further support for previous evidence that abnormally small twitch tensions occurred in the extensor digitorum brevis muscle only when less than $10 \%$ of the motor axons remained (McComas et al., 1971). The mechanism of this functional compensation was not clear from this study but in several subjects with motor unit counts under
100 the size of the remaining mean motor uofie potential size was clearly enlarged. There we्fes other subjects however belonging to the sa group with fewer than 100 motor units in whom the average motor unit potential size was noto increased.

Gutmann has previously shown that the sizes of the motor units of the rat soleus muscle in the older animals are decreased by one-third com $\frac{\mathbb{D}}{2}$ pared with the young. At the same time, there was only slight reduction of the numbers of alpha motor fibres (Gutmann and HanzlíkováJ 1966). Histological studies at the same time showed degenerative changes in the end-plate with nerve terminal regeneration and irregulario ties in the innervation pattern of muscles in old? animals. In a later study (Gutmann, Hanzlíková: and Vyskočil, 1970) Gutmann demonstrated a clear reduction in frequency of miniature end-o plate potentials (MEPP) in older animals, al though the MEPP were increased in size. In that study, there were no fibrillation potentials in the aged rat muscle. 
In the present study there was no evidence of denervation in the form of fibrillation potentials but, unlike the aged rat muscle, the motor unit count was obviously reduced and there was no evidence for a reduction in the size of individual motor units. MEPP frequency is known to be dependent on pre- rather than post-synaptic factors (Katz, 1962) and the reduced frequency of the miniature end-plate potentials may have been early functional signs of primary neuronal failure.

Ellis showed a gradual loss of Purkinje cells in the human cerebellum with age, amounting to a loss of approximately one-third of the Purkinje cells by the age of 90 , compared with the age of 20 years (Ellis, 1920). A loss of similar magnitude in numbers of myelinated fibres in the eighth and ninth thoracic dorsal and ventral nerve roots beginning after the third decade was shown by Corbin (Corbin and Gardner, 1937).

Swallow (1966) clearly showed a substantial reduction in the total fibre content of the anterior tibial nerve with increasing age. In one study of control human peripheral nerves, Wallerian degeneration first appeared at the age of 10 years and increased steadily with age, although segmental demyelination was uncommon until the age of 60 years (Arnold and Harriman, 1970). In the present study, subjects 70 years of age and over had a mean motor unit count less than half that of the subjects 39 years and younger. This was a significantly greater loss than the one-third loss of Purkinje cells or ventral root fibres.

Fullerton has previously shown that in older guinea-pigs the distal transmission latency was prolonged in the median and ulnar nerves and as well, there was a complete or partial loss of myelinated fibres from the median nerve at the wrist (Fullerton and Gilliatt, 1967). It is possible to suggest that, in the present study, part of the reduction of motor unit counts was the result of asymptomatic injury to the median nerve in the region of the carpal tunnel. In all but three subjects, however, the distal latency was less than $4.5 \mathrm{msec}$ and two of the three were over 80 years of age.

In summary, this study has shown a considerable reduction in the number of motor units in control subjects with increasing age. Despite the reduction in the motor unit count, the functional compensation was satisfactory in terms of strength. The cause of the reduction in the motor unit count is not known with confidence but it is likely to be a combination of factors, including asymptomatic injury to the median nerve at the wrist, and primary neuronal cell death.

The author would like to acknowledge the assistance of his wife, Janet, in the statistical analysis of the data and preparation of the draft. The study would not have been possible without the generous cooperation of the author's neurological and neurosurgical colleagues who provided many of the subjects for study.

\section{REFERENCES}

Arnold, N., and Harriman, D. G. F. (1970). The incidence of abnormality in control human peripheral nerves studied by single axon dissection. Journal of Neurology, Neurosurgery, and Psychiatry, 33, 55-61.

Campbell, M. J., and McComas, A. J. (1970). The effects of ageing on muscle function. Fifth Symposium on Current Research in Muscular Dystrophy and Related Diseases, 1970. Abstracts of Communications. Muscular Dystrophy Group of Great Britain: London.

Christensen, E. (1959). Topography of terminal motor innervation in striated muscles from stillborn infants. American Journal of Physical Medicine, 38, 65-78.

Cooper, S. (1960). Muscle spindles and other muscle receptors. In The Structure and Function of Muscle, pp. 381-420. Vol. 1. Edited by G. H. Bourne. Academic Press: New York.

Corbin, K. B., and Gardner, E. D. (1937). Decrease in number of myelinated fibres in human spinal roots with age. Anatomical Records, 68, 63-74.

Davies, D. V., and Coupland, R. E. (Ed.) (1967). Gray's Anatomy, 34 edn., pp. 687-689. Longmans: London.

Eccles, J. C., and Sherrington, C. S. (1930). Numbers and contraction-values of individual motor-units examined in some muscles of the limb. Proceedings of the Royal Society of London, 106, 326-357.

Eccles, R. M., Phillips, C. G., and Chien-Ping, W. (1968). Motor innervation, motor unit organization and afferent innervation of $\mathbf{M}$. extensor digitorum communis of the baboon's forearm. Journal of Physiology, 198, 179-192.

Ellis, R. S. (1920). Norms for some structural changes in the human cerebellum from birth to old age. Journal of Comparative Neurology, 32, 1-33.

Feinstein, B., Lindegaard, B., Nyman, E., and Wohlfart, G. (1955). Morphologic studies of motor units in normal human muscles. Acta Anatomica, 23, 127-142.

Fullerton, P. M., and Gilliatt, R. W. (1967). Median and ulnar neuropathy in the guinea-pig. Journal of Neurology, Neurosurgery, and Psychiatry, 30, 393-402.

Gilliatt, R. W. (1966). Axon branching in motor nerves. In Control and Innervation of Skeletal Muscle, pp. 53-63. Edited by B. L. Andrew. Livingstone: Edinburgh.

Gutmann, E., and Hanzlíková, V. (1966). Motor unit in old age. Nature, 209, 921-922.

Gutmann, E., Hanzliková, V., and Vyskočil, F. (1971). Age changes in cross striated muscle of the rat. Journal of Physiology, 21, 331-343. 
Hopf, H. C. (1962). Untersuchungen über die Unterschiede in der Leitgeschwindigkeit motorischer Nervenfasern beim Menschen. Deutsche Zeitschrift für Nervenheilkunde, 183, 579-588.

Katz, B. (1962). The transmission of impulses from nerve to muscle, and the subcellular unit of synaptic action. Proceedings of the Royal Society of London, B., 155, 455-477.

Kugelberg, E., and Skoglund, C. R. (1946). Responses of single human motor units to electrical stimulation. Journal of Neurophysiology, 9, 391-398.

McComas, A. J., Fawcett, P. R. W., Campbell, M. J., and Sica, R. E. P. (1971a). Electrophysiological estimation of the number of motor units within a human muscle. Journal of Neurology, Neurosurgery, and Psychiatry, 34, 121-131.

McComas, A. J., Campbell, M. J., and Sica, R. E. P. (1971b). Electrophysiological study of dystrophia myotonia. Journal of Neurology, Neurosurgery, and Psychiatry, 34, 132139.
McComas, A. J., Sica, R. E. P., Campbell, M. J., and Upton, A. R. M. (1971). Functional compensation in partially denervated muscles. Journal of Neurology, Neurosurgery, and Psychiatry, 34, 453-460.

McComas, A. J., Sica, R. E. P., and Currie, S. (1971). An electrophysiological study of Duchenne dystrophy. Journal of Neurology, Neurosurgery, and Psychiatry, 34, 461-468.

Rowntree, J. (1949). Anomalous innervation of the hand muscles. Journal of Bone and Joint Surgery, 31-B, 505-510.

Sica, R. E. P., and McComas, A. J. (1971). An electrophysiological investigation of limb-girdle and facioscapulohumeral dystrophy. Journal of Neurology, Neurosurgery, and Psychiatry, 34, 469-474.

Stålberg, E., and Trontelj, J. V. (1970). Demonstration of axon reflexes in human motor nerve fibres. Journal of Neurology, Neurosurgery, and Psychiatry, 33, 571-579.

Swallow, M. (1966). Fibre size and content of the anterior tibial nerve of the foot. Journal of Neurology, Neurosurgery, and Psychiatry, 29, 205-213. 